\title{
Development of a Muon Polarimeter for the T-violation Search Experiment at J-PARC
}

\author{
Aine Kobayashi ${ }^{* a}$,Tatsuo Kawamoto $^{a}$, Jun Imazato ${ }^{b}$, Keisuke Yoshihara ${ }^{a}$, Suguru \\ Shimizu ${ }^{c}$, Youichi Igarashi ${ }^{b}$, Hirohito Yamazaki ${ }^{d}$, Michael Hasinoff ${ }^{e}$, and the TREK \\ collaboration \\ ${ }^{a}$ The University of Tokyo, Hongo, Bunkyo-ku, Tokyo 113-0033, Japan \\ E-mail: aine.kobayashi@cern.ch \\ ${ }^{b}$ KEK, Tsukuba, Japan \\ ${ }^{c}$ Osaka University, Osaka, Japan \\ ${ }^{d}$ Tohoku University, Sendai, Japan \\ ${ }^{e}$ University of British Columbia, Vancouver, BC, Canada
}

\begin{abstract}
TREK is a precision-frontier experiment, planned at J-PARC (Tokai, Japan), for a T-violation search in kaon decays into the $\pi^{0} \mu^{+} v$ final state. The signature is a non-zero transverse polarization $\left(P_{T}\right)$ of muons in the direction perpendicular to the decay plane. Using the same process the E246 experiment at KEK has set an upper limit on $\left|P_{T}\right|<0.0050$ at the $90 \%$ confidence level. TREK is an upgrade of E246 with the goal of achieving more than a factor of 20 higher sensitivity using high intensity Kaon beam from J-PARC and the detector with major upgrades that include a new GEM tracker, new photon device for the CsI(Tl) calorimeter and a new magnet system providing uniform field. The most important element of TREK is the new muon polarimeter of novel design, incorporating an active muon stopper instrumented by an array of drift tubes for tracking. This design allows the tracks of muons and positrons to be reconstructed, providing a large acceptance for positrons with higher analyzing power, background suppression, and for controlling systematic uncertainties such as those arising from uncertainty on the decay position and its distribution. We present an overview of the TREK experiment and detail of the muon polarimeter R\&D with results of studies using Monte Carlo simulation and beam tests of the full size prototype.
\end{abstract}

Technology and Instrumentation in Particle Physics 2014,

2-6 June, 2014

Amsterdam, the Netherlands

* Speaker. 


\section{Introduction}

The TREK experiment [1] will search for violation of time reversal invariance (T-violation) in the $K^{+} \rightarrow \pi^{0} \mu^{+} v_{\mu}\left(K_{\mu 3}\right)$ process at J-PARC, Japan. The transverse muon polarization $P_{T}$ (figure 1) is defined as the asymmetry of the spin component perpendicular to the decay plane $\left(P_{T}=\overrightarrow{\sigma_{\mu}}\right.$. $\left.\left(\overrightarrow{p_{\pi}} \times \overrightarrow{p_{\mu}}\right) /\left|\overrightarrow{p_{\pi}} \times \overrightarrow{p_{\mu}}\right|\right)$, and is a signal of T-violation.

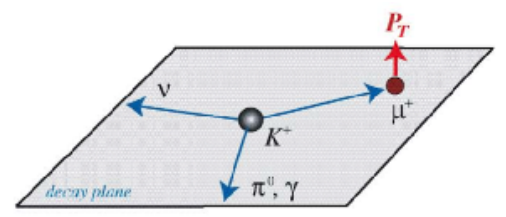

Figure 1: $K_{\mu 3}$ decay and $P_{T}$

There are advantages of using this process such as the very small Standard Model contributions to $P_{T}$ arising from vertex correction $\left(<10^{-7}\right)$ and the smallness of the spurious effects from final state interactions $\left(<10^{-5}\right) . P_{T}$ in the range of $10^{-3} \sim 10^{-4}$ is therefore a sensitive probe for Tviolation, or equivalently CP-violation, beyond the Standard Model. There are theoretical models of new physics that predict a sizable $P_{T}$ without conflicting with other experimental constraints [3]. A previous experiment, KEK-E246 [2] has measured $P_{T}=-0.0017 \pm 0.0023$ (stat) \pm 0.0011 (syst), which is consistent with T-invariance. TREK is an upgrade of E246 with the goal of achieving significantly reduced statistical and systematic errors, by a factor 20 and 10 respectively [4].

\section{TREK experiment}

The basic concept of the experiment, as shown in figure 2, is the same as that of E246: Kaons are stopped in the active fiber target, $\pi^{0} \rightarrow \gamma \gamma$ are detected by the $\mathrm{CsI}(\mathrm{Tl})$ calorimeter, and muons are momentum analyzed by the magnetic spectrometer. Polarization of the muons is finally measured by the muon polarimeter. The muon spectrometer is built around a superconducting iron-core toroidal magnet with 12 equally spaced gaps. Each gap is instrumented by a tracking detector system. TREK uses the high intensity separated $K^{+}$beam of the Hadron Experimental Facility of the J-PARC $50 \mathrm{GeV}$ proton synchrotron. The beam intensity will be about $3 \times 10^{6} \mathrm{kaons} / \mathrm{sec}$ and a total runtime will be $10^{7}$ seconds.

\subsection{Active fiber target}

The target is a bundle of 492 scintillating fibers (figure 3). The active volume is $20 \mathrm{~cm}$ long in the beam direction and $7.5 \mathrm{~cm}$ diameter. The bundle is surrounded by 12 fiducial scintillation counters, each pointing to the corresponding muon spectrometer gap. The TREK fibres are nearly half the size of the E246 fibers. They are readout by Kuraray green WLS fibres glued into a $1 \mathrm{~mm}$ slot machined into 1 side of the $3 \times 3 \mathrm{~mm}^{2}$ square scintillating fiber.

\section{2 $\operatorname{CsI}(\mathrm{Tl})$ calorimeter}

Photons from $\pi^{0}$ decays are detected by a barrel of $768 \mathrm{CsI}(\mathrm{Tl})$ crystals surrounding the fiber target (figure 4). There are 12 holes to let the charged particles enter the spectrometer. The solid 


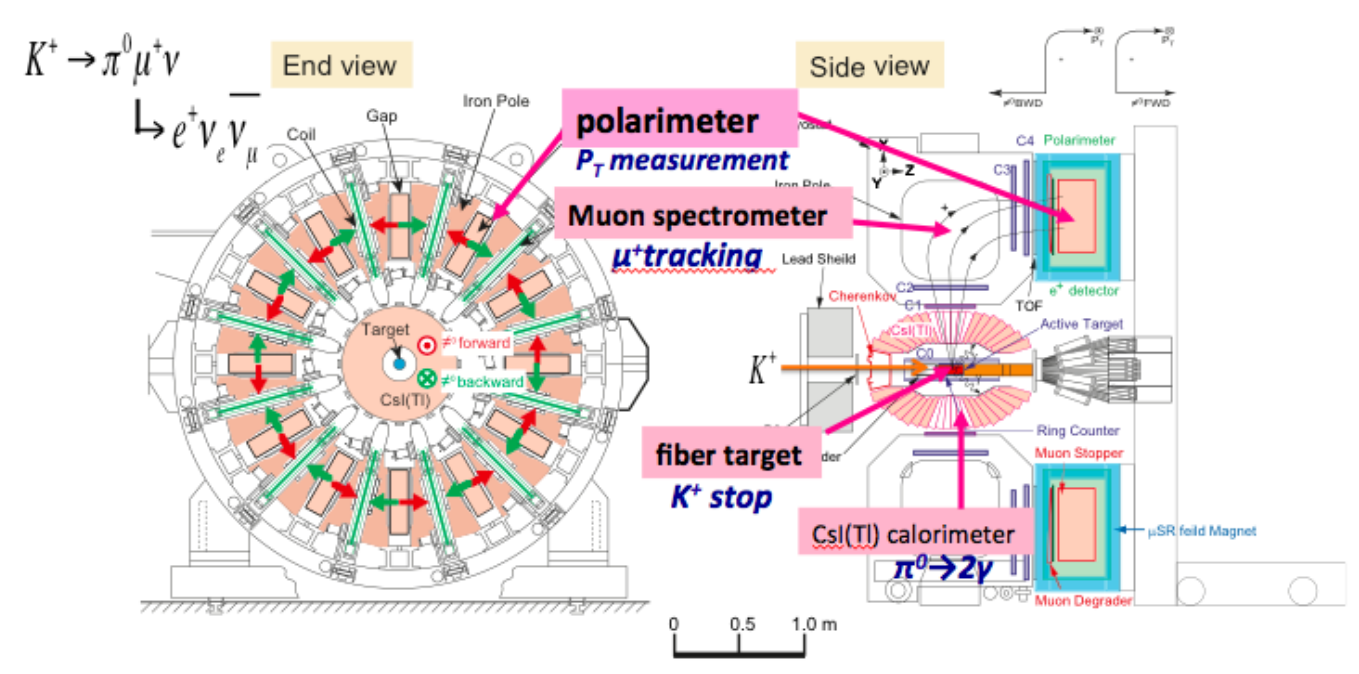

Figure 2: End and side views of the TREK decector sysmtem.

angle coverage is about $3 \pi$ steradian including the acceptance losses due to the beam entrance and exit holes. To improve timing and rate performance, Avalanche Photo Diodes will replace the old photomultipliers, and the signals will be digitized by flash ADCs.

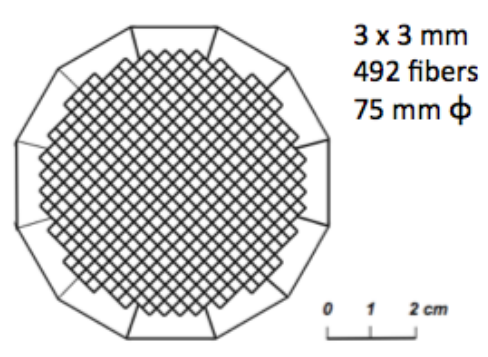

Figure 3: Scintillating fiber target.

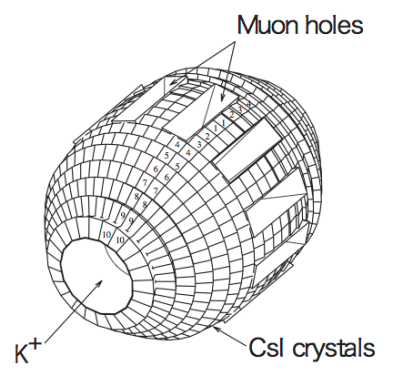

Figure 4: CsI(Tl) barrel with 768 crystal modules.

\subsection{Muon spectrometer}

Good resolution of muon momentum, angle and vertex position are important for accurate determination of the $K_{\mu 3}$ kinematics, rejection of background processes as well as removal of $\mathrm{K}^{+}$ decays in flight. The muon tracking system (figure 5) will be enhanced by adding two new GEM trackers, one $(\mathrm{C} 0)$ close to the kaon stopper and the other $(\mathrm{C} 1)$ in front of the existing tracker $(\mathrm{C} 2)$. In order to reduce multiple scattering, the air gap will be filled by He gas in a large part of the muon tracking volume.

\subsection{Muon polarimeter}

The principle of the polarization measurement is based on the angular asymmetry of $e^{+}$in the decay $\mu^{+} \rightarrow e^{+} v v$ with respect to the muon spin direction. In the E246 experiment, muons were 

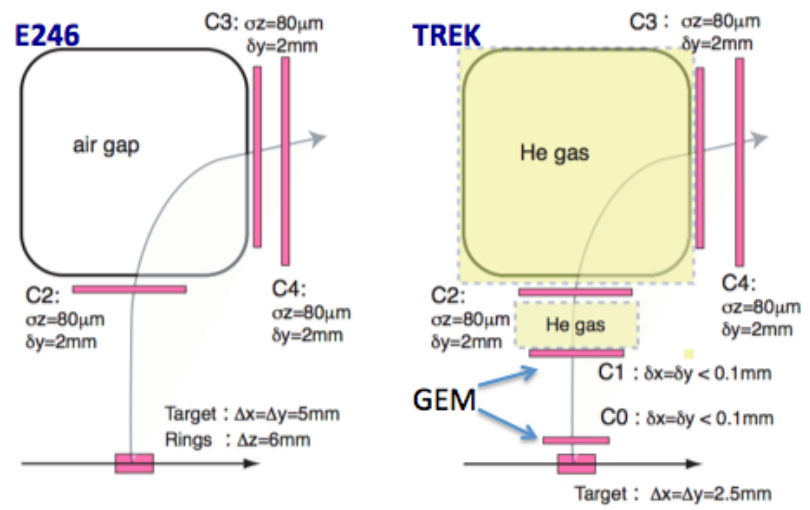

Figure 5: Schematic view of E246 (left) and TREK (right) muon spectrometer.

stopped in a array of aluminum plates, and the positrons were detected by two sets of scintillation counters symmetrically placed both sides (left and right) of the muon stopper (figure 6, left). The acceptance was about $10 \%$ of $4 \pi$. Dominant sources of systematic error were due to uncertainty on the muon stop position and accidental background signals in the $e^{+}$counters. TREK will employ an "active" polarimeter, in which the track of an incoming muon is measured, its stop position determined, and the track of the decay positron is also measured. In this way, the muon decay and its kinematics determined event by event, thus greatly reducing systematic uncertainties.
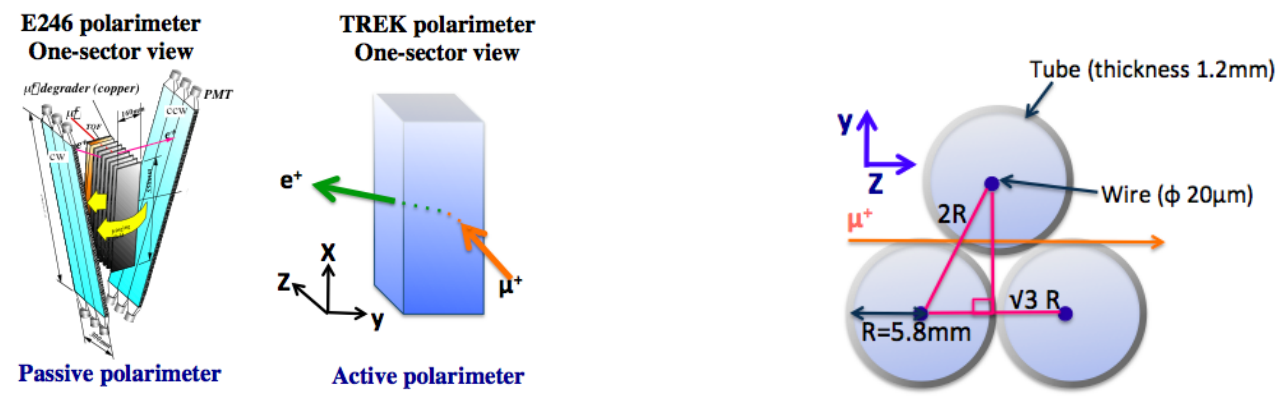

Figure 6: Schematic view of E246 polarimeter (left) Figure 7: Tube layout and dimension of MTP tubes. and TREK polarimeter (right).

\section{Muon Tube Polarimeter (MTP)}

The muon tube polarimeter (MTP) is an array of 371 drift tubes made of $1.2 \mathrm{~mm}$ thick aluminum (figure 7). The dimension of the units are $865 \mathrm{~mm}(x) \times 284 \mathrm{~mm}(y) \times 330 \mathrm{~mm}(z)$. The tube diameter is $14 \mathrm{~mm}$. The anode is a stainless steel wire of $20 \mu \mathrm{m}$ diameter. The aluminum wall works as the muon stopper. The thickness is chosen to effectively stop the muons from the $K_{\mu 3}$ decays while maintaining the multiple scattering at an acceptable level for positron tracking. The wire signals are readout from both ends and signal timing and charge are digitized. The coordinates in the plane perpendicular to the wires $(z-y)$ are measured using the position of hit wire and drift 
time while the coordinate parallel to the wires $(x)$ is determined by charge division. A full size prototype has been built and tested.

\subsection{Beam test of the prototype}

The MTP prototype was tested at TRIUMF using the M11 beam line. Figure 8 shows the experimental set up. The momentum of the beam was set at $170 \mathrm{MeV}$. Coincidence of the two scintillation counters, S0 and S2, defined the timing of incoming particle and used to trigger data readout. Separation of muons from pions was performed by Time Of Flight. In front of the MTP was a degrader made of aluminum. The thickness was adjusted $(3.25 \mathrm{inch}$ ) so that the muons stop at the center of the MTP volume. Figure 9 shows the distribution of muon stop positions. Wire signals were readout using CAEN V792 QDC, CAEN V1190B TDC. Most of the data were taken with readout from one side of the wires, measuring $z-y$ coordinates only. Due to the limitation of available electronics channels, the four white corners shown in figure 9 were not readout. A dedicated measurement was done with readout electronics attached to both ends of the wires for a study of charge division performance. Polarization of the beam muons was measured using the

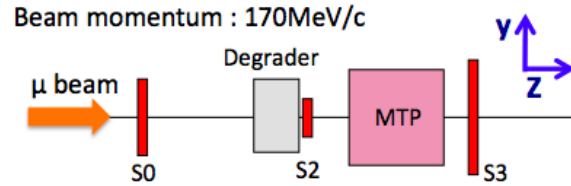

Figure 8: Setup for the MTP beam test.

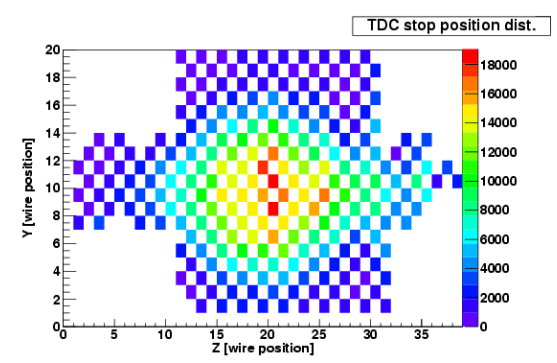

Figure 9: The distribution of muon stop position in $z-y$ plane of MTP.

$\mu$ SR technique at the same place after the MTP test [5]. Figure 10 shows the precession pattern. The polarization was found to be $17 \%$ to the backward $(-z)$ direction.

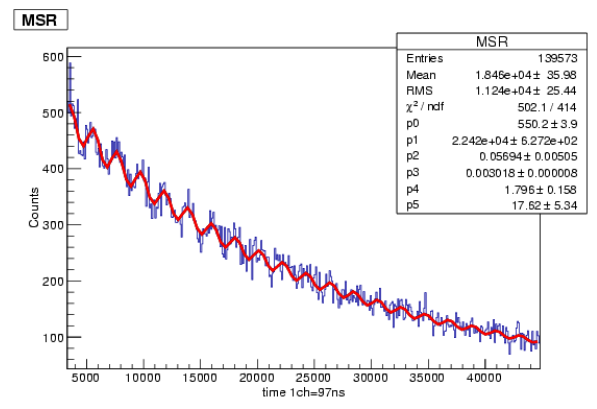

Figure 10: The $\mu$ SR oscillation spectrum.

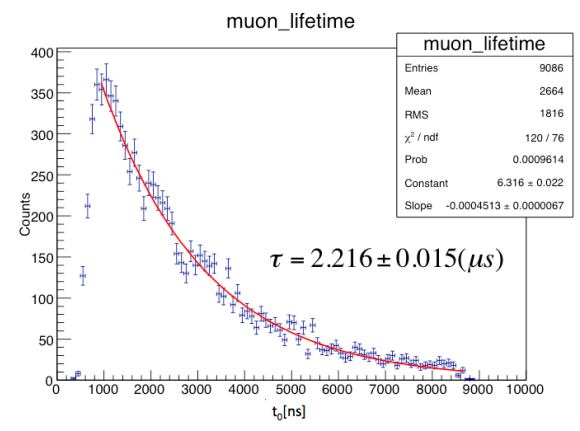

Figure 11: The fitting results of $e^{+}$timing.

\subsection{Drift tube analysis}

The maximum drift time is about $90 \mathrm{~ns}$. The $x$ - $t$ relation has been derived from the spectrum and expressed by a 2nd order polynomial. Hits are identified as positron hits if they are more than 
$250 \mathrm{~ns}$ later than the muon arrival time $\left(t_{0}\right)$. A track fit by straight line to the drift circles with its time origin as a free parameter provides the timing of positron decay (figure 11). A fit to an exponential curve yields decay constant that is in agreement with the muon lifetime.

\section{Track reconstruction for polarization analysis}

Muon polarization was analyzed based on the decay angle distribution projected in the 2-d ( $z$ $y$ ) plane defined by the tube coordinates (figure 12). The angle $\psi$ is the angle between the incoming muon direction $(+z)$ and the decay positron, and is related to the $3-\mathrm{d}$ polar angle $\theta$ and azimuthal angle $\phi$ by :

$$
\cos \psi=z / \sqrt{z^{2}+y^{2}}=\cos \theta / \sqrt{\cos ^{2} \theta+\left(1-\cos ^{2} \theta\right) \sin ^{2} \phi} .
$$

The observed distribution of $\cos \psi$ is a result of an average over the angle $\phi$. In the track reconstruction, muon and positron hits are each fitted to a straight line. A simple approach is adopted using only the position of hit wires with the uncertainty on 'drift radius' set to the (tube diameter) $\sqrt{12}$ in the $\chi^{2}$ fit; this is justified by considering the multiple scattering of comparable size. At least three hit tubes are required. The muon stop fiducial volume is set at the center of MTP in a rectangular region of $7 \times 6$ tubes.

The distribution of $\cos \psi$ is shown in figure 13 and compared to a Monte Carlo simulation based on GEANT4 [6] generated with muon longitudinal polarization of $-17 \%$. There are spikes at $\cos \psi \sim 0$ and \pm 0.5 reflecting the regular structure of the tube array. The Monte Carlo simulation reproduces the distribution including the spikes.

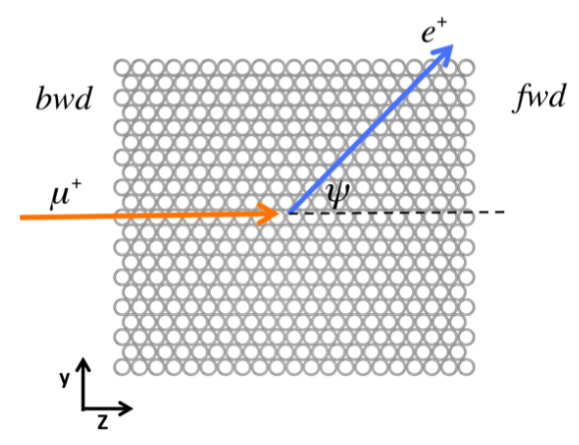

Figure 12: Schematic view of the muon decay into positron in the MTP.

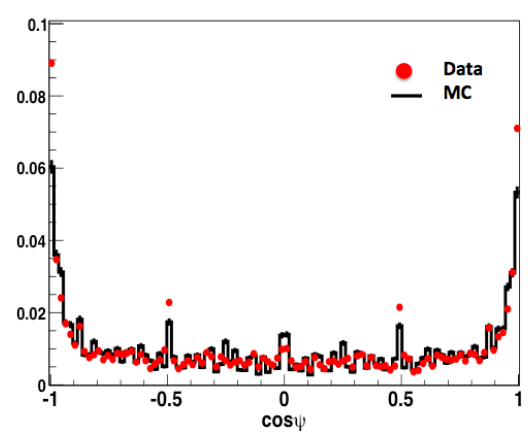

Figure 13: Comparison of the beam test data with $\mathrm{MC}$ simulation.

Reconstruction efficiency was studied using the Monte Carlo simulation. Figure 14 (a) shows the positron track efficiency as a function of positron energy. The efficiency reaches plateau of 70$80 \%$ above $1 / 2$ of the maximum positron energy $\left(E_{\max }\right)$. The efficiency loss at low energy is due to shorter track length and larger scattering of low energy positrons. Since higher energy positrons are better collimated to the muon spin direction (no correlation below $1 / 2 E_{\max }$ ) this efficiency curve is favorable for polarization measurement. Figure 14 (b) shows the positron efficiency as a function of polar angle $\theta$. The dip around 0 is due to the fact that the positrons pass through smaller number of tubes when the track is parallel to the tubes, i.e. $\phi=0$ or $\pi$. This is seen in figure 14 (c) where the efficiency is plotted as a function of $\phi$. 


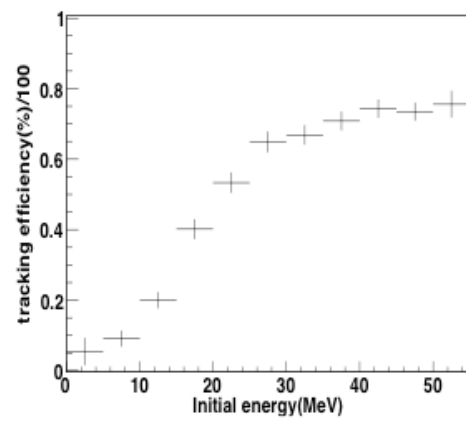

(a)

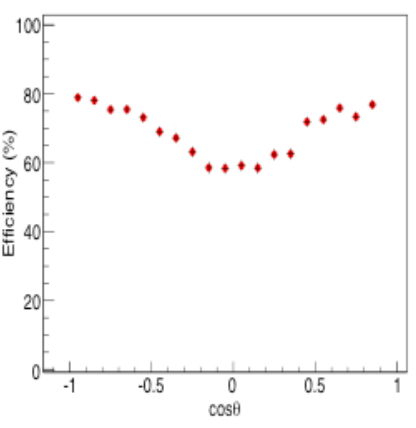

(b)

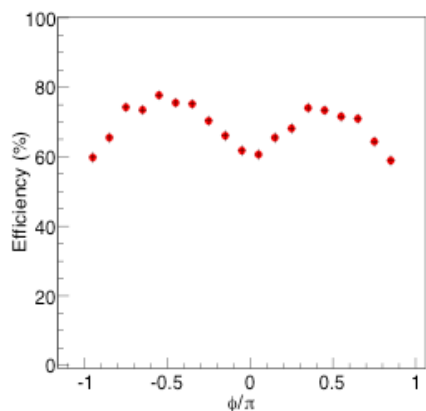

(c)

Figure 14: The positron efficiency as a function of energy (a), $\cos \theta(b)$ and $\phi(c)$.

\section{Determination of muon polarization}

The MTP data are used to determine the polarization of the beam muons. An unbinned maximum likelihood fit is used. Probability density function (PDFs) of positron angle for $+100 \%$ and $-100 \%$ polarization, each denoted respectively by $F_{1}$ and $F_{2}$, are produced by the Monte Carlo simulation and expressed as normalized histograms. The distribution of angular variable $x$ is given by :

$$
F(x, p)=\frac{1}{2}(1-p) F_{1}(x)+\frac{1}{2}(1+p) F_{2}(x),
$$

here the fitting parameter $p$ is the polarization. In constructing the PDF, care is taken to ensure the normalization is independent of polarization $p . F_{1}(x)$ is a sum of histogram for $+100 \%$ and flipped $(x \rightarrow-x)$ histogram for $-100 \%$, and similarly for $F_{2}(x)$. In this way, the relation $F_{1}(x)=F_{2}(-x)$ holds, ensuring that $F(x, p)$ is independent of $p$.

The muon polarization is analyzed for the longitudinal $(z)$ and transverse $(y)$ component using the distribution of $\cos \psi$ and $\sin \psi$, respectively. The expected statistical uncertainty $\sigma\left(P_{T}\right)$ is $\sim 5 / \sqrt{N}$ where $N$ is the number of events. In order to see any biases arising from instrumental asymmetry, data were taken with four different settings of MTP; (1) standard, (2) rotated to flip forward-backward, (3) rotated to flip up and down, and (4) both forward-backward and up-down. Results of polarization fits are shown in figure 15. Longitudinal polarization for the position (1) is $-20.5 \pm 1.0 \%$, which is consistent with the $\mu$ SR measurement. The longitudinal polarization changes sign when MTP is rotated to swap forward and backward (positions 2 and 4), as expected. Transverse polarization at position (1) is about $5 \%$. If this is the result of small polarization in transverse direction, it should change sign when MTP is rotated upside down (position 3). However actual change is not as expected, $7.9 \pm 1.1 \%$ and $8.8 \pm 1.4 \%$ for position (3), (4). This observation suggests the presence of small bias, in particular in the small transverse component.

\subsection{Possible biases}

Positron tracking efficiency is not symmetric between upward and downward direction when the muon stop position is close to the upper or lower edge of MTP, since the available number 
of tubes is different in the two hemispheres. Exact symmetry of tracking efficiency is only for the muons stopping at the center of MTP. In order to see the dependence of apparent polarization arising from efficiency asymmetry, value of fitted transverse polarization is shown in figure 16 as a function of vertical stop position in 7 horizontal slices within the fiducial volume. Position 4 corresponds to the nominal center, where the value of transverse polarization is $3.5 \pm 2.7 \%$. A position dependent bias exists. However, in the TREK experiment, the definition of sign of

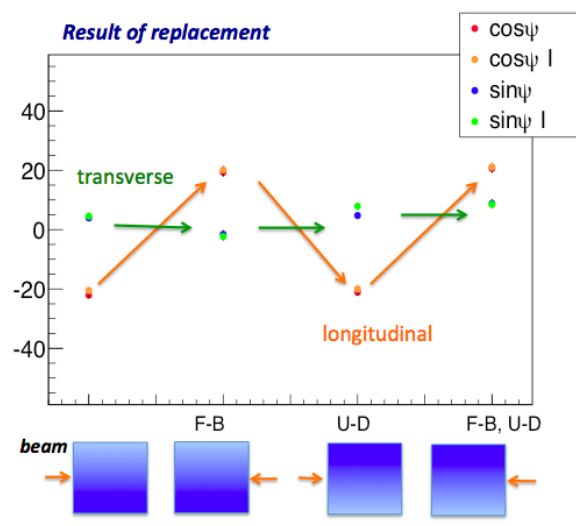

Figure 15: The results of the polarization fit.

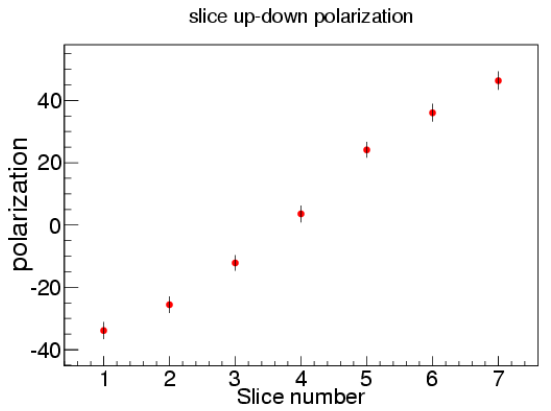

Figure 16: The fitting results of the each slices.

transverse polarization depends on the direction of $\pi^{0}$ at the $K^{+}$decay; for forward and backward $\pi^{0}$ the direction of positive transverse polarization is opposite, therefore position dependent bias will average out to zero if the forward and backward $\pi^{0}$ events are equally used. In addition, event by event determination of the muon stop position allows monitoring such cancellation takes place.

\section{Summary}

The muon polarimeter plays a important role in the measurement of $P_{T}$. The performance of the polarimeter was studied by using the data of the beam test and Monte Carlo simulation.

Measured longitudinal asymmetry is consistent with the $\mu$ SR result. The statistical error $\sigma_{P_{T}}$ was $5 / \sqrt{N}$. Monte Carlo simulation results reproduced the data well.

\section{References}

[1] TREK homepage, http://trek.kek.jp/

[2] M. Abe et al. [E246 collaboration], Phys. Rev. D 73, 072005 (2006)

[3] V. P. Efrosinin et al, Phys. Lett. B493, 293 (1997); R. Garisto and G. Kane, Phys. Rev. D44, 2038 (1991); M. Fabbrichesi and F. Vissani, Phys. Rev. D55, 5334 (1997)

[4] TREK/E06 proposal (2006), http://j-parc.jp/NuclPart/pac_0606/pdf/p06-Imazato_2.pdf

[5] A. Kobayashi, Development of Muon Polarimeter for J-PARC T-violation search experiment, Master's Thesis, The University of Tokyo (2012)

[6] A. Agostinelli et al., Nucl. Instrum. Meth. A, 2003, 506, 250 